\title{
\begin{tabular}{l|l} 
Mibraries & DSpace@MIT
\end{tabular}
}

\author{
MIT Open Access Articles
}

\section{Joined Probabilistic Load Flow and Sensitivity Analysis of Distribution Networks Based on Polynomial Chaos Method}

The MIT Faculty has made this article openly available. Please share how this access benefits you. Your story matters.

Citation: Gruosso, Giambattista et al. "Joined Probabilistic Load Flow and Sensitivity Analysis of Distribution Networks Based on Polynomial Chaos Method." IEEE Transactions on Power Systems 35, 1 (January 2020): 618 - 627. (C) 2020 IEEE

As Published: http://dx.doi.org/10.1109/tpwrs.2019.2928674

Publisher: Institute of Electrical and Electronics Engineers (IEEE)

Persistent URL: https://hdl.handle.net/1721.1/130949

Version: Final published version: final published article, as it appeared in a journal, conference proceedings, or other formally published context

Terms of use: Creative Commons Attribution-Noncommercial-Share Alike 


\title{
Joined Probabilistic Load Flow and Sensitivity Analysis of Distribution Networks based on Polynomial Chaos Method
}

\author{
Giambattista Gruosso, Roberto S. Netto, Luca Daniel, and Paolo Maffezzoni
}

\begin{abstract}
Due to the statistical uncertainty of loads and power sources found in smart grids, effective computational tools for probabilistic load flow analysis and planning are now becoming indispensable. In this research, we describe a unified simulation framework that allows quantifying the probability distributions of a set of observation variables as well as evaluating their sensitivity to potential variations in the power demands. The proposed probabilistic technique relies on the generalized Polynomial Chaos algorithm and on a region-wise aggregation/description of the time-varying load profiles. It is shown how detailed statistical distributions of some important figures of merit, which includes voltage unbalance factor in distribution networks, can be calculated with a two-orders of magnitude acceleration compared to standard Monte Carlo analysis. In addition, it is highlighted how the associated sensitivity analysis is of guidance for the optimal allocation and planning of new loads.
\end{abstract}

Index Terms-Polynomial Chaos method, Probabilistic load flow, Sensitivity analysis, Unbalanced networks, Uncertainty quantification.

\section{INTRODUCTION}

Smart distribution grids are expected to provide new types of services, e.g. charging of electrical vehicles, while exploiting new forms of distributed power generation, e.g. higher penetration of renewable energy sources. An increasing active role of consumers is also envisaged: they will be able to alter their usage patterns in order to follow the trends of electricity prices [1].

Distribution network evolution and the management of the bidirectional power flow will require a large number of monitoring devices able to provide data/information about the status of the network and the quality of service at several points. A massive deployment of (smart) meters and monitoring systems is thus expected in the near future [2]-[5], moving from a situation where little information is known to a situation where data is available at many points. This will allow achieving a better understanding of the network performance and will enable the use of statistical techniques to characterize and predict the comprehensive behavior of the grid. Even the power flow will no longer be directed in a single prevailing direction, but it will have a greater variability due to the presence of storage systems or electric vehicles that represent bidirectional loads.

In this context, a relevant role is also envisaged for novel

G. Gruosso and P. Maffezzoni are with Politecnico di Milano, DEIB, I20133 Milan, Italy, R. S. Netto is with FIT Instituto de Tecnologia,Sorocaba, Sao Paulo, Brazil, L. Daniel is with Massachusetts Institute of Technology (MIT), Cambridge, MA 02139, USA. robust/efficient simulation tools for probabilistic analysis and planning [6], [7]. In fact, uncertainty-aware computational tools can provide a comprehensive view of the overall network in the presence of uncertain power demand and generation, e.g. they can predict bus voltages and line currents statistical variations at network points that can hardly be measured. Furthermore, these techniques can be used for the analysis of the sensitivity with respect to the direction of the power flow, starting from the grid description and a limited set of measurement.

Probabilistic Load Flow (PLF) analysis methods consist in using probabilistic models for the power loads as well as in replacing deterministic load flow simulation with proper stochastic analysis methods. The basic and reference stochastic method remains Monte Carlo (MC) simulation even though it can be computationally demanding due to the great numbers of samples it requires to represent uncertain inputs. MC simulation combined with advanced sampling methods [8] can alleviate the computational burden for statistical problems of small size.

Efficient analytical/approximate stochastic techniques have been recently investigated in the field of power systems [9]-[13]. Among them, the point estimate method [9], [10] provides approximations of the raw statistical moments of some observation variables, while the cumulant method [11], [12] works for linear (or almost linear) problems. In this paper, instead, we will focus on the category of Polynomial Chaos (PC) methods [13] since such techniques work for nonlinear problems and provide the detailed Probability Density Function (PDF) of the desired observation variables.

The polynomial chaos method has been recently employed to efficiently derive the PDF and cumulative probability of line voltages by considering constant power loads at a fixed time instant [13].

In this paper, we build on these recently presented techniques as well as on generalized Polynomial Chaos (gPC) Theory [14] to extend its application/usage. In our analysis, we consider realistic load power profiles, described by quasi static time series (QSTS) over given observation time windows, and we aggregate the profiles in areas, or geographic regions, of the network infrastructure.

The original contributions of the proposed method could be summarized in the following issues:

1. We propose a region-wise uncertainty quantification analysis where load uncertainty in each region and for each one of the three phase lines is represented by a single in- 
dependent random variable. This approach allows dealing with the relevant case of 1-phase loads.

2. We exploit the gPC paradigm to evaluate the PDF of a set of Quantities of Interest (QoIs) that affect the quality of the network. Such QoIs can include the peak and minimum voltage at some observation nodes, and over some observation time windows, as well as the peak value of other figures of merit such as the Voltage Unbalance Factor (VUF).

3. The last issue considered in this paper is connected with the analysis of the impact that new loads can have on the grid. The analysis aims at identifying the regions where new loads insertion could be useful, e.g for voltage balancing. The increase or decrease in power demand at a given line phase in one of the regions, for instance due to the allocation of new 1-phase loads, reverberate on the nodal voltages in other regions (and phase lines) in a way that is not easy to be predicted. This is the case when a storage system is integrated in the grid, or in general a stochastically dependent load or source is considered: therefore operational planning and proper allocation of new loads require region-wise and phasewise sensitivity analysis tools able to efficiently foresee the effects that variations in the aggregated loads of a region can have on the whole network. Several numerical methods for sensitivity analyses in power distribution lines have been provided in the literature that exploit the Jacobian matrix used in power flow calculation [15] or adopt a perturb and observe approach [16]. The first category of techniques are intrusive methods that need the access to the simulation code. In this paper, we concentrate on the second category of methods. We show how an efficient region-wise sensitivity analysis can be naturally derived by exploiting the same gPC simulation framework used for PLF. As a result, the PLF analysis and region-wise sensitivity can be implemented in a homogeneous framework.

The remainder of this paper is organized as follows: in Sec. II we shortly review the deterministic power flow problem and illustrate, with an example, the aims and importance of variability analysis. In Sec. III, the region-wise approach is outlined, while in Sec. IV the gPC-based uncertainty quantification method and sensitivity analysis are described. In Sec. $\mathrm{V}$, we provide more details about the $\mathrm{gPC}$ implementation in connection with the Stochastic Testing (ST) selection of sampling points. Finally, in Sec. VI we report simulation results for a IEEE benchmark distribution network case study. In particular, we prove how the proposed methodology can be of guidance for the optimal allocation of new loads in the network.

\section{Power Demand Variability and Motivation of THE WORK}

For the sole purpose of demonstrating the application of the method, a low-voltage IEEE test network is used, mainly consisting of three-phase or single-phase loads. In addition, the low-voltage grid helps to define quality factors with which it is possible to demonstrate the validity of the proposed approach even in unbalanced three-phase simulations. The methodology can be extended without any substantial modification to networks powered at higher voltages or when there are power sources. In the following, the considered low voltage distribution network is made of $N_{l}$ lines and $N$ buses and designed to provide the prescribed power flows at the network terminals. Deterministic load flow analysis is mathematically formulated as a set of nonlinear equations of the type:

$$
\mathbf{F}_{n}(\overrightarrow{\mathbf{V}})=\mathbf{S}_{n}-\mathbf{V}_{n} \sum_{i=1}^{N} \mathbf{Y}_{n i} \mathbf{V}_{i}^{*}=\mathbf{0}
$$

for $n=1, \ldots, N$. In (1), $\mathbf{S}_{n}=P_{n}+j Q_{n}$ denotes the complex power injection at node $n$ where $P_{n}$ and $Q_{n}$ are the active and reactive powers respectively at network terminations. $\mathbf{V}_{n}$ denotes the $n$th node voltage phasor, while $\mathbf{Y}_{n i}$ are the entries of the bus admittance matrix. Node voltage phasors are collected into vector $\overrightarrow{\mathbf{V}}$. Power demand at terminations vary in time and thus powers $P_{n}(t), Q_{n}(t)$ are functions of time. For a given observation time window (e.g. a day or a week), that is discretized into a sequence of $N_{t}$ equally-spaced time instants $t_{m}=m \cdot \Delta t$, power demand is thus specified as given power profiles $P_{n}^{0}\left(t_{m}\right), Q_{n}^{0}\left(t_{m}\right)$ for $m=1, \ldots, N_{t}$. Node voltage waveforms $\mathbf{V}_{n}\left(t_{m}\right)$ are thus calculated for such nominal load conditions by repeatedly solving the nonlinear problem (1) over the sequence of time instants $t_{m}$ using the simulation platform OpenDss [17]. This software performs quasi-static time series (QSTS) simulations, i.e. the chronology of loads at adjacent time points is accounted for by enforcing the dependency of the solution on the history of loads.

Due to the uncertainty of power demand, actual power profiles exhibit variations around their nominal values that can be described statistically. Such a statistical variability of power loads induces fluctuations in the node voltages that may bring them out of the safe limits and compromise the quality of the service. The peak and minimum magnitude values assumed by node voltages over some observation time windows are thus crucial QoI in order to check if the network is operating properly.

In our analysis, and in the results presented in Sec. VI, we will consider 3-phase distribution networks with 1-phase loads connected to the three-phase lines. In this case, the power demand variation of the total load connected to a given phase line can affect the node voltages on the three phase lines with fluctuations in the peaks and minima that are difficult to be predicted a priori. Node voltage fluctuations can depend on power load variations in a nonlinear way and with different sign (i.e. positive or negative) on the three phase lines thus introducing possible voltage unbalance. Such a problem for instance occurs when charging electrical vehicles in residential dwelling [18].

\section{REgion-Wise Probabilistic AnAlysis}

The method that we propose in this paper is independent of the simulation scenario, the loads behavior and the grid topology. However, for the sake of illustration, we focus on an example based on Low Voltage distribution grids. In our 


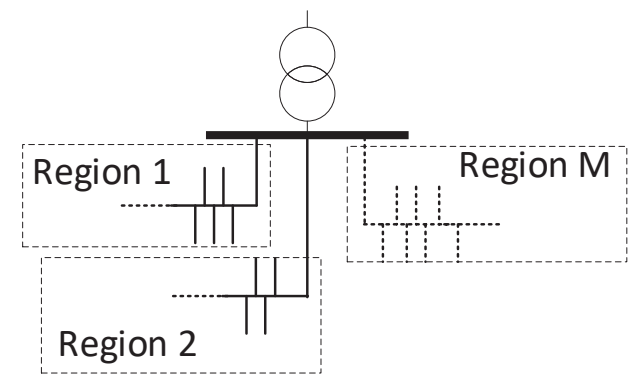

Fig. 1. Example of subdivision of the grid into M Regions, each one of them containing loads connected to the 3 phases.

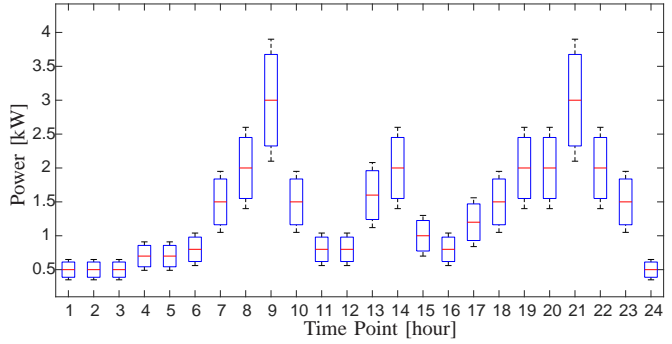

Fig. 2. Example of load variation produced by the model (2). In each time instant, it is reported the mean value (i.e. the nominal load profile) and the standard deviation.

probabilistic analysis, we suppose to partition the network into $M$ disjunct geographic regions $\mathrm{R}_{k}$, with $k=1, \ldots, M$ as shown in Fig. 1. Each region represents an aggregation of loads, e.g., the loads of the same building or block. However, other choices may be considered as well.

The active power profiles $P_{n_{i}}(t)$ at all of the nodes $n_{i}$ internal to a given region $\mathrm{R}_{k}$, i.e. $n_{i} \in \mathrm{R}_{k}$, and connected to a given phase line are modeled in the following way:

$$
P_{n_{i}}(t)=p_{n_{i}}^{0}(t)\left[1+\sigma_{n_{i}} \xi_{r}\right]
$$

where $p_{n_{i}}^{0}(t)$ is the known nominal power profile at node $n_{i}$. In (2), $\xi_{r}$ is a zero-mean unitary-variance random variable described by the PDF $\rho_{r}\left(\xi_{r}\right)$ that incorporates the power demand uncertainty (in the frame of uncertainty quantification such a random variable is commonly referred to as a parameter uncertainty). In this paper, we will mainly focus on Gaussiandistributed random variables $\xi_{r}$ since this is the type of load model most frequently derived by field measurements [19]. However, it is worth observing how the gPC method illustrated in the next Section allows handling statistical parameters with several nonGaussian statistical distributions, as described in [14], and complex combinations of them [20]. The random variable $\xi_{r}$ produces a distribution (i.e., either a reduction or increase) of the actual active power $P_{n_{i}}(t)$ around the nominal profile $p_{n_{i}}^{0}(t)$. Fig. 2 shows an example of load variation produced by the model described in (2). The quantity $\sigma_{n_{i}}$ is a scaling constant that determines the degree of uncertainty at node $n_{i}$. In general, two nodes aggregated in the same region may have different degrees of uncertainty. In this way, the active power $P_{n_{i}}(t)$ is a stochastic process whose mean value and standard deviation are given by [21]:

$$
\begin{aligned}
& \left\langle P_{n_{i}}(t)\right\rangle=p_{n_{i}}^{0}(t) \\
& \sqrt{\left\langle\left(P_{n_{i}}(t)-p_{n_{i}}^{0}(t)\right)^{2}\right\rangle}=\sigma_{n_{i}} p_{n_{i}}^{0}(t) .
\end{aligned}
$$

It is worth observing that for a network partitioned in $M$ regions, the number $l$ of independent statistical parameters $\xi_{r}$ is $l=3 \times M$. By changing $M$ it is possible to vary the detail of the analysis from the simple case with a single region (and 3 statistical parameters modulating total loads at each phase) to the extreme case where the power demand at each single (1-phase) load is weighted by an independent statistical parameter.

While power demands (2) represent the inputs of the probabilistic analysis, the outputs are given by a set of $q$ observation variables, that are expected to affect the quality of service, generically denoted as $V^{j}$, with $j=1, \ldots, q$. In this case the set of q observation variables are specifically chosen voltages, but they could be every Quantity of Interest.

Such variables can include the peak and minimum values assumed by the phase voltages (or line currents) over some observation time windows. For the case of 1-phase loads, which is considered in this paper, another relevant observation variable is the peak value of the VUF. The percentage VUF is defined as the ratio of the negative voltage sequence component $V_{n}$ to the positive voltage sequence component $V_{p}$ [22], i.e.

$$
\mathrm{VUF}=\frac{\left|V_{n}\right|}{\left|V_{p}\right|} \cdot 100
$$

\section{UnCERTAINTY QUANTIFICATION AND SENSITIVITY ANALYSIS WITH GPC METHOD}

\section{A. Uncertainty Quantification}

We formalize the probabilistic problem where the uncertain load power profiles are described by means of $l$ random Gaussian-distributed variables $\xi_{r}$ as in (2). Such quantities are collected into the vector $\vec{\xi}=\left[\xi_{1}, \xi_{2}, \ldots, \xi_{l}\right]$. In view of (2), each realization of the random variables $\xi_{r}$ corresponds to well determined power profiles and thus to well determined voltage profiles calculated by solving the load flow problem (1). As a result, the $j$ th observation variable $V^{j}(\vec{\xi})$ (e.g. a node voltage) is a nonlinear function of the random variables $\vec{\xi}$ and thus it is a random variable as well.

The generalized polynomial chaos (gPC) method consists in approximating each observation variable with an order- $\beta$ truncated series expansion of the type [14]

$$
V^{j}(\vec{\xi}) \approx \sum_{i=0}^{N_{b}-1} c_{i}^{j} H_{i}(\vec{\xi})
$$

formed by $N_{b}$ multi-variate basis functions $H_{i}(\vec{\xi})$ weighted by unknown polynomial chaos coefficients $c_{i}^{j}$.

Each multi-variate basis function is given by the product

$$
H_{i}(\vec{\xi})=\prod_{r=1}^{l} \phi_{i_{r}}\left(\xi_{r}\right)
$$

where $\phi_{i_{r}}\left(\xi_{r}\right)$ is a univariate orthogonal polynomial of degree $i_{r}$ [23]. The form of the univariate polynomials depends on 
the density function of the $r$ th parameter $\xi_{r}$. For Gaussiandistributed variables $\xi_{r}$, the associated $\phi_{i_{r}}\left(\xi_{r}\right)$ are the Hermite polynomials

$$
\begin{aligned}
\phi_{0}\left(\xi_{r}\right) & =1 \\
\phi_{1}\left(\xi_{r}\right) & =\xi_{r} \\
\phi_{2}\left(\xi_{r}\right) & =\xi_{r}^{2}-1 \\
\phi_{3}\left(\xi_{r}\right) & =\xi_{r}^{3}-3 \xi_{r}
\end{aligned}
$$

The PC polynomials satisfy the orthogonality property

$$
\left\langle\phi_{i}, \phi_{j}\right\rangle=\int_{\mathbb{R}} \phi_{i}\left(\xi_{r}\right) \phi_{j}\left(\xi_{r}\right) \rho_{r}\left(\xi_{r}\right) d \xi_{r}=\delta_{i, j},
$$

where $\langle\cdot, \cdot\rangle$ denotes the scalar product operator, and $\delta_{i, j}$ is the Kronecker delta function.

For a given number of parameters $l$ and series expansion truncation order $\beta$, the degrees $i_{r}$ of univariate polynomials in (6) forming $H_{i}(\vec{\xi})$, for $r=1, \ldots, l$, satisfy the following relation

$$
\sum_{r=1}^{l} i_{r} \leq \beta .
$$

As a consequence, for truncation order $\beta$ and number of parameters $l$, the number of $\mathrm{gPC}$ basis functions in (5) is given by [23]

$$
N_{b}=\frac{(\beta+l) !}{\beta ! l !} .
$$

Once the coefficients $c_{i}^{j}$ are computed, the mean value and standard deviation of the $j$ th observation variable $V^{j}(\vec{\xi})$ can easily be deduced [23]. In addition, the gPC expansion (5) provides a surrogate compact model for the multi-dimensional relationship $V^{j}(\vec{\xi})$ that links observation variables to random Gaussian parameters. The compact gPC model (5) can then be used in connection with the MC method in order to determine the detailed PDF shape of $V^{j}(\vec{\xi})$. This is achieved by generating a very large number $N_{m c}$ of uncertainty vectors $\vec{\xi}^{k}=$ $\left[\overrightarrow{\xi_{1}^{k}}, \overrightarrow{\xi_{2}^{k}}, \ldots, \overrightarrow{\xi_{l}^{k}}\right]$, i.e. the realizations, accordingly to the joint probability distribution of variables in $\vec{\xi}$. For each realization $\vec{\xi}^{k}$, the corresponding realization of the observation variable $V^{j}(\vec{\xi})$ is evaluated by means of (5) in a numerically efficient way (much more efficiently than running a LF analysis). As the number $N_{m c}$ of evaluations grows, at limit tending to infinity, the distribution of values calculated with the gPC model tends to the statistical distribution of $V^{j}(\vec{\xi})$. As a result, the detailed PDF shape of $V^{j}(\vec{\xi})$ can be determined in very short times, i.e. one million evaluations take a few seconds on a quad-core computer.

\section{B. Sensitivity Analysis}

For notational simplicity, we denote $V(\vec{\xi})=V^{j}(\vec{\xi})$ the $j$ th observation variable while, in order to further explain the meaning of the gPC formulation presented in the previous subsection, we restrict to the case of expansion order $\beta=2$ and $l=3$ random parameters. For such a case, the gPC expansion (5) of $V(\vec{\xi})$ subject to the constraint (9) reads

$$
\begin{aligned}
V\left(\xi_{1}, \xi_{2}, \xi_{3}\right) & \approx c_{0}+c_{1} \xi_{1}+c_{2} \xi_{2}+c_{3} \xi_{3} \\
& +c_{4} \xi_{1} \xi_{2}+c_{5} \xi_{1} \xi_{3}+c_{6} \xi_{2} \xi_{3} \\
& +c_{7}\left(\xi_{1}^{2}-1\right)+c_{8}\left(\xi_{2}^{2}-1\right)+c_{9}\left(\xi_{3}^{2}-1\right) .
\end{aligned}
$$

The sensitivity of $V(\vec{\xi})$ versus the $r$ th parameter $\xi_{r}$ results:

$$
\frac{\partial V(\vec{\xi})}{\partial \xi_{r}} \mid \vec{\xi}=\overrightarrow{0}=c_{r}
$$

and thus it simply corresponds to the $r$ th linear term coefficient in the gPC expansion.

\section{Computing the GPC COefFicients}

There are two mainstream approaches for computing the gPC expansion coefficients in (5): Galerkin Projection (GP) and Collocation Method (CM) [24]. Galerkin projection is an intrusive numerical technique that requires modifying the LF code (1). GP is numerically robust, however the formation and solution of the projection equations require a significant computational effort which limits the practical applicability to problems of small size and with a few statistical parameters (23 ). For such reasons, in this paper we will focus on Collocation methods.

\section{A. Stochastic Collocation}

Stochastic collocation, is an approximate technique that allows the application of gPC method to problems with a greater number of statistical parameters. Furthermore, Stochastic collocation is a non-intrusive and thus it can be combined with any LF formulation (1) without modifying the implementation codes. According to collocation method, a gPC expansion of the type (5) is adopted for each observation variables $V^{j}$. Then, the expansion coefficients $c_{i}^{j}$ in the series (5) are calculated by properly selecting a set of testing points where the series expansions (5) are enforced to fit the values of observation variables $V_{k}^{j}$. Stochastic collocation is very efficient however its accuracy depends on the way testing points are selected. In this paper, we will focus on a recentlyproposed robust method referred to as Stochastic Testing (ST) method which allows implementation as a non intrusive collocation method [23]. According to ST method the $N_{b}$ unknown coefficients $c_{j}$ in the series (5) are calculated by selecting $N_{s}=N_{b}$ testing points $\vec{\xi}^{k}$, for $k=1, \ldots, N_{s}$ with the method outlined in the next Sec. V. In each one of the testing points, the observation variable $V_{k}(t)=V\left(\vec{\xi}^{k}\right)$ is evaluated by running a deterministic LF analysis. Hence, the series expansions (5) are enforced to fit exactly (i.e., the polynomials interpolate the samples) the values $V_{k}^{j}$ at the testing points. For the $j$ th observation variable, this results in the following linear system

$$
\mathbf{M} \vec{c}^{j}=\vec{V}^{j},
$$

where $\vec{c}^{j}=\left[c_{1}^{j}, \ldots, c_{N_{b}}^{j}\right]^{T}$ and $\vec{V}^{j}=\left[V_{1}^{j}, \ldots, V_{N_{s}}^{j}\right]^{T}$ are the column vectors collecting the unknown coefficients and observation variable values respectively. 
The $N_{b} \times N_{b}$ square matrix $\mathbf{M}=\left\{a_{k, i}\right\}=\left\{H_{i}\left(\vec{\xi}^{k}\right)\right\}$ collects the gPC basis functions evaluated at the testing points, i.e.

$$
\mathbf{M}=\left[\begin{array}{ccc}
H_{0}\left(\vec{\xi}^{1}\right) & \ldots & H_{N_{b}-1}\left(\vec{\xi}^{1}\right) \\
\vdots & \ddots & \vdots \\
H_{0}\left(\vec{\xi}^{N_{s}}\right) & \ldots & H_{N_{b}-1}\left(\vec{\xi}^{N_{s}}\right)
\end{array}\right]
$$

It is worth observing that matrix $\mathbf{M}$, sometimes referred to as the experiment matrix, remains the same for each observation variable, so it is precalculated, inverted and used for any $j$ as follows:

$$
\vec{c}^{j}=\mathbf{M}^{-1} \vec{V}^{j} .
$$

Generalizations of (15) have also been presented in the literature where a number of testing points greater than the number of basis is selected, i.e. $N_{s}>N_{b}$, [25].

In this case, the overdetermined system (15) can be solved with a linear regression technique such as the least-squares method:

$$
\vec{c}^{j}=\left(\mathbf{M}^{T} \mathbf{M}\right)^{-1} \mathbf{M}^{T} \vec{V}^{j} .
$$

\section{B. Testing points selection}

According to gPC+ST method, the selection of the testing points $\vec{\xi}^{k}$ in the stochastic space is done as to preserve its robustness compared to Galerkin projection method. To this aim, the scalar product (that implements projection) between two polynomials of the series expansion (i.e. the product of such polynomials has degree $\beta$ at most) is best approximated by a Gauss quadrature formula with $\beta+1$ nodes

$$
\left\langle\phi_{i}, \phi_{j}\right\rangle=\int_{\mathbb{R}} \phi_{i}\left(\xi_{r}\right) \phi_{j}\left(\xi_{r}\right) \rho_{r}\left(\xi_{r}\right) d \xi_{r} \approx \sum_{k=1}^{\beta+1} \phi_{i}\left(\xi_{r}^{k}\right) \phi_{j}\left(\xi_{r}^{k}\right) w_{r}^{k},
$$

where $\xi_{r}^{k}$ denotes the $k$ th quadrature node and $w_{r}^{k}$ the corresponding weight. The $\beta+1$ quadrature nodes $\xi_{r}^{k}$ are thus good testing points for the single uncertainty parameter $\xi_{r}$ (i.e. the univariate problem). As an example, for a Gaussian-distributed variable $\xi_{r}$ and expansion order $\beta=3$, the $\beta+1=4$ quadrature nodes are given by

$$
\xi_{r}^{1}=-2.33, \quad \xi_{r}^{2}=-0.74, \quad \xi_{r}^{3}=0.74, \quad \xi_{r}^{4}=2.33
$$

and the associated weights are

$$
w_{r}^{1}=0.046, \quad w_{r}^{2}=0.45, \quad w_{r}^{3}=0.45, \quad w_{r}^{4}=0.046 .
$$

When the multivariate case with $l$ parameters is concerned, the testing points vectors $\vec{\xi}^{k}=\left[\xi_{1}^{k}, \xi_{2}^{k}, \ldots, \xi_{l}^{k}\right]$ are determined by considering the multi-dimensional grid of all of the possible combinations (i.e. the tensor product) of the univariate quadrature nodes. In the case of two random variables $\xi_{1}, \xi_{2}$, for instance, and 4 univariate quadrature nodes as in (18), the nodes in the two-dimensional grid are $4^{2}=16$ and include the following $\left(\xi_{1}, \xi_{2}\right)$ value couples

$$
\begin{aligned}
& (-2.33,-2.33),(-2.33,-0.74),(-2.33,0.74),(-2.33,2.33), \\
& (-0.74,-2.33),(-0.74,-0.74), \quad \ldots
\end{aligned}
$$

Each point in the multi-dimensional grid has also associated a Gauss weight which is given by the product of the related

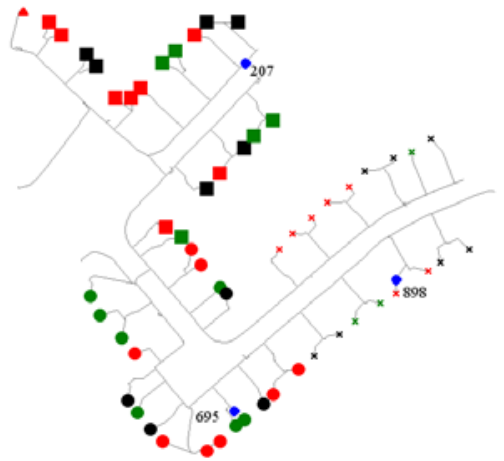

Fig. 3. Topology of the IEEE LV European test feeder. Nodes $(207,695,898)$ in three different regions are monitored in order to determine the effect of the load variation. Mark legend: square $=$ Region I, Circle $=$ Region II, cross $=$ Region III. Color Legend: Red=Phase A, Black=Phase B, Green $=$ Phase C.

univariate weights. In the example (20) the associated weigths are

$$
\begin{array}{lllll}
0.002, & 0.02, & & 0.02, & 0.002, \\
0.02, & 0.2, & \ldots & &
\end{array}
$$

respectively. In general, the number $(\beta+1)^{l}$ of nodes in the multi-dimensional grid is greater than the number $N_{b}$ of basis functions defined in (10). A subset of quadrature nodes can thus be selected as testing points to form systems (15) or (16). Stochastic testing selection strategy consists in preferring those quadrature nodes associated to largest Gauss weights and leading to the best (smallest) condition number for the experiment matrix $\mathbf{M}[23]$.

\section{NUMERICAL RESULTS}

In this section, we focus on low voltage networks having radial topology where the impact of load unbalance is expected to be particularly significant. However, the proposed analysis is general and can easily be extended to other types of electrical systems and other topologies, such as looped or weakly looped ones. The considered network is the IEEE European low voltage test feeder [26] described by the circuit reported in Fig. 3 that represents a benchmark case study. This test feeder is radial with a base frequency of $50 \mathrm{~Hz}$, at $230 \mathrm{~V}$ (phase voltage)/416 $\mathrm{V}$ (line to line voltage). The medium voltage system supplying the substation is modeled as a voltage source with an impedance (Thevenin equivalent) according with [26]. The three-phase network has 906 low voltage nodes that are connected by 905 branches. The value of the line impedance and shunt admittance used are defined in [26], but due to the short length of lines (the branches are shorter than one hundred meters) the shunt admittance is neglected and just the series impedance is considered. The original benchmark is provided with 55 1-phase loads that are applied to the nodes represented by marks in Fig. 3 and subdivided as follows: 21 for the phase A (red), 19 for the phase B (Black), 15 for the phase C (Green).

\section{A. Probabilistic analysis}

The proposed region-wise uncertainty quantification analysis is applied by partitioning the 55 given loads into three 
TABLE I

NUMber of LOADS FOR REgIONS AND PHASES

\begin{tabular}{||c|c|c||c|c|c||c|c|c||}
\hline \hline I-A & I-B & I-C & II-A & II-B & II-C & III-A & III-B & III-C \\
\hline 7 & 7 & 5 & 7 & 5 & 7 & 7 & 7 & 3 \\
\hline \hline
\end{tabular}

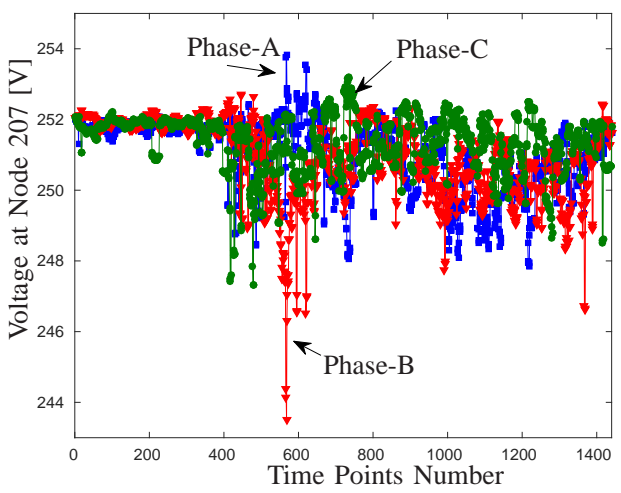

Fig. 4. Time domain evolution of the three phases at node 207: (Square Marker) Phase A, (Triangle Marker) Phase B, (Circle Marker) Phase C.

geographic regions as shown in Fig. 3: Region I contains 19 loads (square mark in Fig. 3) distributed among the three phase lines as reported in Table I, Region II has 19 loads (circle mark) while Region III includes 17 loads (cross mark).

In what follows, load flow simulations are performed using quasi-static time series power profiles made of 1440 samples (i.e., 24 hours $\times 60$ minutes) that change for the different loads.

Fig. 4 reports the waveforms of the three phase voltages at node 207, used here as the monitoring node for Region I, simulated with OpenDSS with the nominal load profiles provided with the benchmark. Voltage waveforms exhibit sharp fluctuations in time, from 6:00 A.M. on, due to the variations in the load profiles. A first set of QoI is thus represented by the peaks and minima values assumed by the phase voltages over given observation time windows. As an example, we consider the time window from 9:00 A.M to 10:00 A.M. (corresponding to samples from 540 to 600 ) and with the proposed gPC+ST method, we calculate the statistical distribution of the minima and peak values, assumed over the time window, for the three phase voltages, A, B and C. To this aim, the uncertainty in the load profiles is modeled as in (2) by means of $l=9$ zeromean, unitary-variance Gaussian distributed parameters $\xi_{r}$ that randomly scale the given nominal power profiles. The degree of uncertainty at each node $n_{i}$ is fixed to $\sigma_{n_{i}}=0.2$ for all of the loads and phase lines.

In view of (10), for $l=9$ stochastic parameters and $\mathrm{gPC}$ expansion order $\beta=2$, the gPC series expansion is made of 55 basis functions. In our implementation we generate 56 testing points in the space of parameters using the stochastic testing method reviewed in Sec. V.A and for each one of them a deterministic load flow solution is performed. The extra sample point is used in a leave-one-out cross validation error method to check the accuracy of the gPC expansion with $\beta=2$. Fig. 5 shows, as an example, the curve provided by

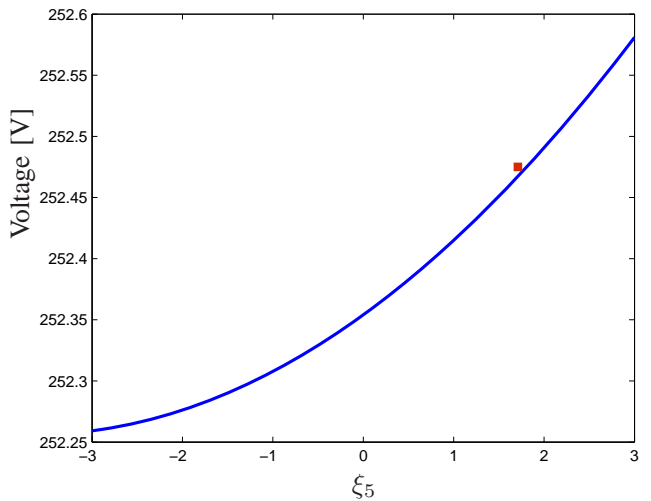

Fig. 5. The line represents the curve of the Phase-C peak value provided by th $\mathrm{gPC}$ model vs $\xi_{5}$ with the other $\xi_{r}$ equal to 0 . The red square Marker represents the extra sample used for verification.

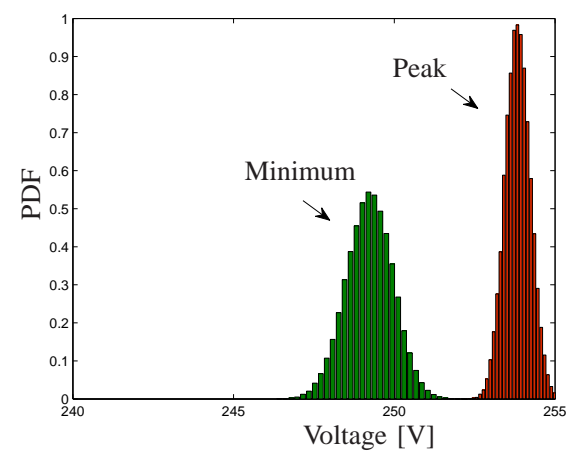

Fig. 6. (Histogram) Statistical distribution of the minimum and peak values for the Phase A at node 207 on the 9:00-10:00 time window.

the gPC model for one observation variable considered in what follows (i.e. the peak value of the Phase-C at node 207) as a function of one of the parameter uncertainty (i.e. parameter $\xi_{5}$ ) with the other parameters fixed to zero. The red square marker represents the extra sample (i.e. not used to calculate the gPC model). Two observations are in order: (i) the peak voltage dependence versus parameter $\xi_{5}$ is truly nonlinear; (ii) the gPC model with truncation order $\beta=2$ provides a good fitting at the extra sample meaning that, for the example considered, expansion order $\beta=2$ is adequate.

Figs. 6, 7, and 8 show the calculated PDF for the three phases A, B, C, respectively at node 207 .

We see how, for the load arrangement provided by the benchmark, Phase-B reaches the lowest voltage levels and exhibits the greatest uncertainty in the minimum value that ranges within the interval $(242,245) \mathrm{V}$ with $90 \%$ probability. By contrast, Phase A reaches the highest voltage levels with the peak value that ranges within the interval $(253.5,255) \mathrm{V}$ with $90 \%$ probability. Finally, Phase C fluctuates within narrower intervals but its peak value is non-Gaussian distributed. This result can be better seen with the aid of Fig. 9 where the statistical distributions of the peak value of Phase-C computed with the proposed gPC and with the reference MC method are reported and compared with the Gaussian distribution of equal mean value and variance.

The reference MC method uses 10,000 runs (i.e. deter- 


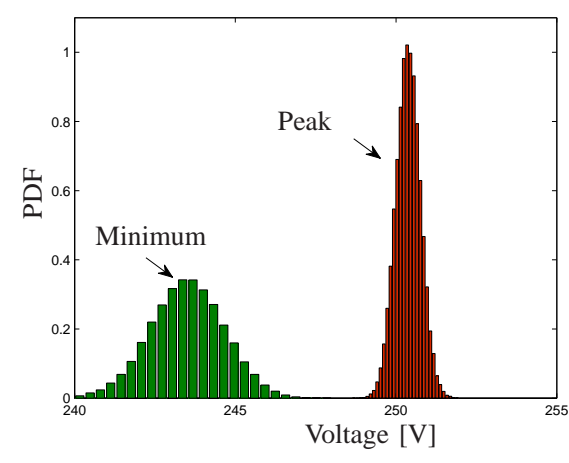

Fig. 7. (Histogram) Statistical distribution of the minimum and peak values for the Phase B at node 207 on the 9:00-10:00 time window.

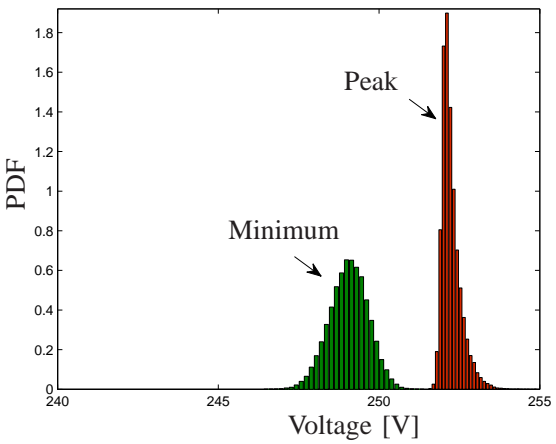

Fig. 8. (Histogram) Statistical distribution of the minimum and peak values for the Phase $C$ at node 207 on the 9:00-10:00 time window.

ministic load flow Solutions) selected with a latin-hypercube sampling. With this setting, the peak value distributions provided by the proposed $\mathrm{gPC}$ and reference MC method are almost superimposed and the associated standard deviations, i.e. $\sigma_{g P C}=0.3080 \mathrm{~V}$ and $\sigma_{M C}=0.3071 \mathrm{~V}$ respectively, match within a relative accuracy of $2 \%$. Since the gPC+ST method only requires 55 deterministic analyses, it introduces a $180 \times$ computational speedup factor compared to the reference $\mathrm{MC}$ analysis for the same accuracy. Considering that the computational effort is due mainly to the OpenDss simulation of the QSTS that takes around 5 seconds, the speed up factor is proportional to the number of samples. Table II reports the standard deviations predicted by the MC method for a growing number of samples and the relative error compared to the value provided by the reference MC (i.e. with 10,000 samples). If a lower order of accuracy for MC method, e.g. a $5 \%$ accuracy (which requires about 6000 samples), is accepted the computational cost of $\mathrm{MC}$ remains about 100x greater than that of gPC. It is thus reasonable to say that, for our example, gPC results to be about two orders of magnitude faster than MC.

As a further check, we also use the point estimate scheme described in [9], which adopts a numerically efficient samples selection method, to estimate the raw moments of the output variables of interest. The standard deviation predicted by the numerically-efficient point estimate method for the peak value of Phase-C is $\sigma_{P E M}=0.225 \mathrm{~V}$ so that the relative error compared to reference MC analysis is about $27 \%$.

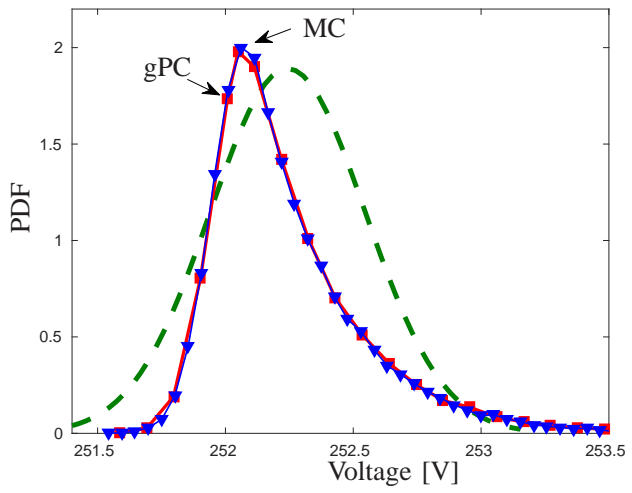

Fig. 9. Detail of the distributions of the Phase-C peak value as computed with: (Red Square Marker) gPC and (Blue Triangle Marker) MC method (with 10, 000 samples). (Green Dashed Line) Gaussian distribution of equal mean value and variance.

TABLE II

CONVERGENCE OF MC VS NUMBER OF SAMPLES

\begin{tabular}{||l||l|l|l||}
\hline \hline Number of Samples & 2500 & 5000 & 10000 \\
\hline$\sigma_{M C}$ & 0.351 & 0.324 & 0.3071 \\
\hline error & $14 \%$ & $5.5 \%$ & - \\
\hline $\begin{array}{l}\text { CPU time for } 1440 \text { point timeserie [sec] } \\
\text { Intel Core i5 }-3.20 \mathrm{GHz}\end{array}$ & 12500 & 25000 & 50000 \\
\hline \hline
\end{tabular}

\section{B. Sensitivity analysis: a strategy for load guidance}

The probability distributions of the peaks and minima for the three phases at a given observation node provide a measure of their statistical uncertainty over the considered observation time window. Phase voltage uncertainty corresponds to an analog statistical uncertainty in the voltage unbalance factor VUF defined in (4) which represents another relevant QoI. The statistical distribution of the VUF can be derived with the proposed gPC+ST method by using the same 55 deterministic load flow Solutions described in the previous subsection VI.A. Furthermore, according to (12), the linear coefficients of the gPC expansion for the VUF observation variable provide detailed information about its sensitivity with respect to possible variations of the power demands in each region and phase line.

Table III reports the sensitivity coefficients for the three observation nodes 207, 695 and 898 in the three Regions. For a compact notation, such values are reported multiplied by a factor 100. A first important information contained in Table III is the sign of the sensitivity coefficients: a negative sign indicates that an increase in power demand, at that phase and Region, corresponds to a decrease of the VUF and thus to a beneficial effect on load balancing. Viceversa, a positive sign of the sensitivity coefficient indicates that an increase in power demand will move the network towards greater unbalance.

In order to check this result, we repeat the probabilistic analysis with 10 new loads, randomly selected among those provided with the benchmark, connected to the distribution network. Two different allocation strategies, referred to as Case a) and Case b) and described in Tables IV and Tables V, respectively, are investigated. The allocation of Case a) is done by exploiting the information provided by the gPC-based sensitivity analysis and concentrates the new loads in the Regions 
TABLE III

VUF SENSITIVITY COEFFICIENTS $\times 100$

\begin{tabular}{||l||c|c|c||c|c|c||c|c|c||}
\hline \hline & I-A & I-B & I-C & II-A & II-B & II-C & III-A & III-B & III-C \\
& $\xi_{1}$ & $\xi_{2}$ & $\xi_{3}$ & $\xi_{4}$ & $\xi_{5}$ & $\xi_{6}$ & $\xi_{7}$ & $\xi_{8}$ & $\xi_{9}$ \\
\hline N. 207 & -0.25 & 3.48 & -0.82 & -0.65 & 6.98 & -0.25 & -0.31 & 5.79 & -0.08 \\
\hline N. 695 & -0.13 & 3.68 & -1.08 & -1.02 & 12.18 & -0.51 & -0.41 & 9.19 & -0.14 \\
\hline N. 898 & -0.11 & 3.7 & -1.13 & -0.98 & 12.22 & -0.54 & -0.47 & 11.63 & -0.15 \\
\hline \hline
\end{tabular}

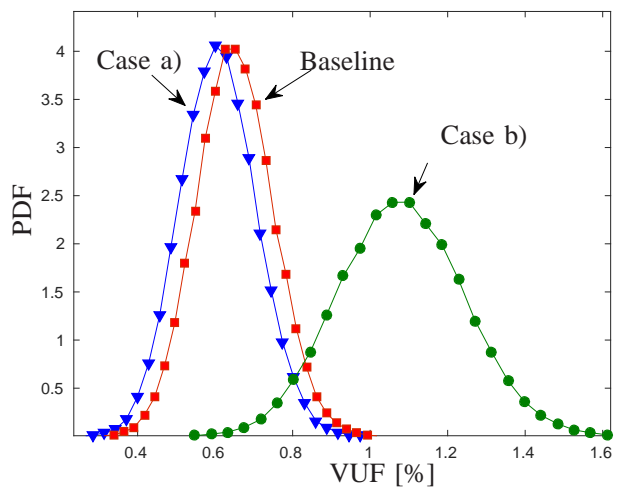

Fig. 10. Statistical distribution of the VUF at node 207 .

and phases having negative sensitivity coefficients with large module. Viceversa, allocation of Case b) is done in defiance of sensitivity analysis, i.e. putting new loads in the Regions and phases with large positive sensitivity coefficients. Figs. 10, 11,12 show the statistical distributions of the maximum VUF, computed over the time window 9:00-10:00 A.M., for the two different allocation cases and the three observation nodes. It is apparent how Case b) results in a balance deterioration: the mean value and the standard deviation of the VUF increase significantly. At node 898, the allocation of Case b) leads to a $40 \%$ probability that the peak VUF will exceed the upper bound of $2 \%$. A similar violation is seen for VUF at node 695 . By contrast, the allocation of Case a) results in a reduction of the mean value and standard deviation of the VUF in all of the three observation nodes compared to the baseline case (i.e. 55 loads distributed as in Table I). This shows how sensitivity analysis can help allocating new loads while preserving, or even improving, load balancing in the network.

TABLE IV

Number of LOADS FOR CASE A)

\begin{tabular}{||c|c|c||c|c|c||c|c|c||}
\hline \hline I-A & I-B & I-C & II-A & II-B & II-C & III-A & III-B & III-C \\
\hline 7 & 7 & 8 & 11 & 5 & 7 & 10 & 7 & 3 \\
\hline \hline
\end{tabular}

TABLE V

NUMBER OF LOADS FOR CASE B)

\begin{tabular}{||c|c|c||c|c|c||c|c|c||}
\hline \hline I-A & I-B & I-C & II-A & II-B & II-C & III-A & III-B & III-C \\
\hline 7 & 10 & 5 & 7 & 9 & 7 & 7 & 10 & 3 \\
\hline \hline
\end{tabular}

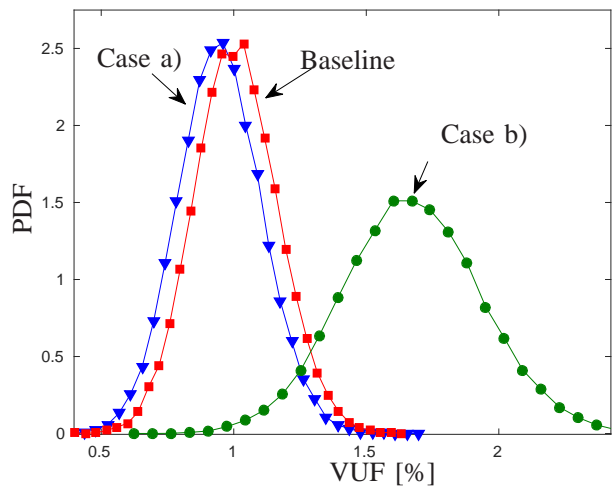

Fig. 11. Statistical distribution of the VUF at node 695.

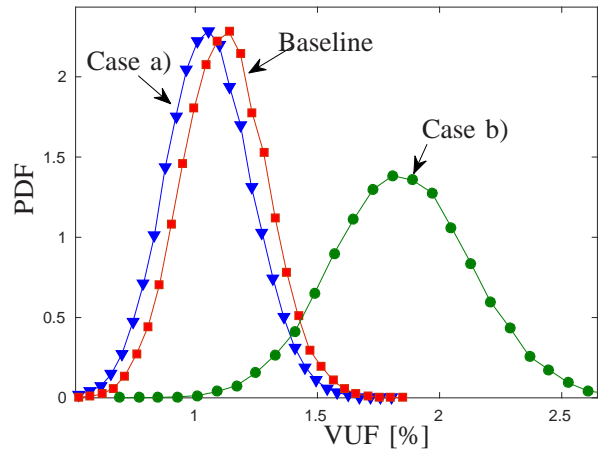

Fig. 12. Statistical distribution of the VUF at node 898 .

We conclude our analysis by recalculating the VUF at node 898 in the baseline case and in the load re-allocation Cases a), b) when the uncertainty of load profiles in (2) is modelled by nonGaussian distributed random variables $\xi_{r}$. In particular, we show the results when the 9 random variables $\xi_{r}$ are all uniformly distributed into the interval $[-1,1]$. This case is shown in Fig. 13. Comparing Fig. 13 with Fig. 12 we see how changing the load profiles distribution does not affect the final qualitative result, i.e. load reallocation a) improves the VUF while reallocation b) results in a significant VUF deterioration. We conclude that the loads reallocation strategy based on gPC and sensitivity analysis works well independently of the statistical distribution of the load profiles.

\section{CONCLUSION}

In this paper, we have described a unified computational framework for probabilistic load flow and sensitivity analyses in distribution networks with uncertain load profiles. Our approach relies on a region-wise uncertainty quantification analysis that aggregates loads within geographic regions of the network and on the usage of the generalized Polynomial Chaos method. We have shown how the detailed PDF of several QoI affecting the quality of service can be calculated with a speed-up factor of $\approx 100 \times$ compared to standard Monte Carlo analysis for the same accuracy. In particular, the numerical results have been focused on evaluating the statistical distribution of node voltage peak and minima over 


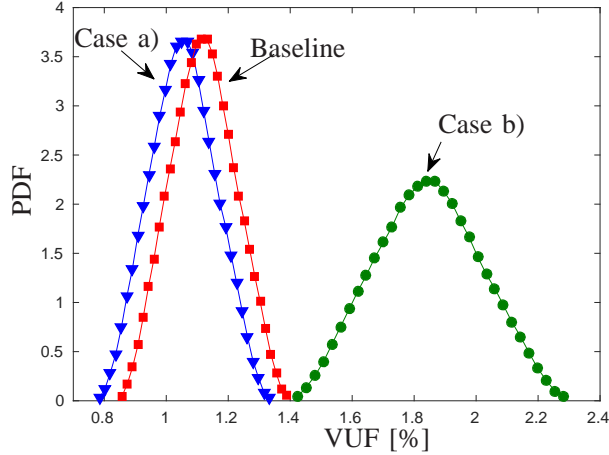

Fig. 13. Statistical distribution of the VUF at node 898 in the case of uniformly-distributed power loads.

a given observation time window and on voltage unbalance factor. Finally, we have proved with an example how the proposed sensitivity analysis can be exploited to properly allocate new (1-phase) loads in the network while preserving, or even improving, load balancing. The proposed methodology relies on a non-intrusive Polynomial Chaos technique that can be combined with any code implementing power flow analysis. As a result, the method can be mixed with advanced unbalanced distribution power flow techniques that integrate the information provided by remote terminal units and achieve high efficiency by breaking the grid topology into simpler subsystems [5]. In future works, we plan to extend the method so as to include storage systems that behave as loads or generators and renewable sources.

\section{REFERENCES}

[1] K. Jhala, B. Natarajan, and A. Pahwa, "Probabilistic voltage sensitivity analysis (pvsa)a novel approach to quantify impact of active consumers," IEEE Transactions on Power Systems, vol. 33, no. 3, pp. 2518-2527, May 2018.

[2] F. Valle, V. Klonari, T. Lisiecki, O. Durieux, F. Moiny, and J. Lobry, "Development of a probabilistic tool using monte carlo simulation and smart meters measurements for the long term analysis of low voltage distribution grids with photovoltaic generation," International Journal of Electrical Power \& Energy Systems, vol. 53, pp. 468 - 477, 2013.

[3] S. Mihai, G. Lipari, A. Monti, A. Voulkidis, G. Zanetto, A. Corsi, L. Toma, G. Fiorentino, and D. Federenciuc, "Next generation realtime smart meters for ict based assessment of grid data inconsistencies," Energies, vol. 10, p. 857, 062017.

[4] M. Neaimeh, R. Wardle, A. M. Jenkins, J. Yi, G. Hill, P. F. Lyons, Y. Hbner, P. T. Blythe, and P. C. Taylor, "A probabilistic approach to combining smart meter and electric vehicle charging data to investigate distribution network impacts," Applied Energy, vol. 157, pp. 688 - 698, 2015.

[5] C.-W. Ten and Y. Tang, Electric Power: Distribution Emergency Operation, 1st ed. CRC Press, 2018.

[6] Y. Wang, N. Zhang, Q. Chen, J. Yang, C. Kang, and J. Huang, "Dependent discrete convolution based probabilistic load flow for the active distribution system," IEEE Transactions on Sustainable Energy, vol. 8, no. 3, pp. 1000-1009, July 2017.

[7] J. Tang, F. Ni, F. Ponci, and A. Monti, "Dimension-adaptive sparse grid interpolation for uncertainty quantification in modern power systems: Probabilistic power flow,' IEEE Transactions on Power Systems, vol. 31, no. 2, pp. 907-919, March 2016.

[8] M. Hajian, W. D. Rosehart, and H. Zareipour, "Probabilistic power flow by monte carlo simulation with latin supercube sampling," IEEE Transactions on Power Systems, vol. 28, no. 2, pp. 1150-1159, May 2013.

[9] J. M. Morales and J. Perez-Ruiz, "Point estimate schemes to solve the probabilistic power flow," IEEE Transactions on Power Systems, vol. 22, no. 4, pp. 1594-1601, Nov 2007.
[10] G. Plattner, H. F. Semlali, and N. Kong, "Analysis of probabilistic load flow using point estimation method to evaluate the quantiles of electrical networks state variables," CIRED - Open Access Proceedings Journal, vol. 2017, no. 1, pp. 2087-2091, 2017.

[11] M. Fan, V. Vittal, G. T. Heydt, and R. Ayyanar, "Probabilistic power flow studies for transmission systems with photovoltaic generation using cumulants," IEEE Transactions on Power Systems, vol. 27, no. 4, pp. 2251-2261, Nov 2012.

[12] P. Amid and C. Crawford, "A cumulant-tensor-based probabilistic load flow method," IEEE Transactions on Power Systems, vol. 33, no. 5, pp. 5648-5656, Sept 2018.

[13] Z. Ren, W. Li, R. Billinton, and W. Yan, "Probabilistic power flow analysis based on the stochastic response surface method," IEEE Transactions on Power Systems, vol. 31, no. 3, pp. 2307-2315, May 2016.

[14] D. Xiu and G. E. Karniadakis, "The wiener-askey polynomial chaos for stochastic differential equations," SIAM Journal on Scientific Computing, vol. 24, no. 2, pp. 619-644, 2002.

[15] F. Tamp and P. Ciufo, "A sensitivity analysis toolkit for the simplification of mv distribution network voltage management," IEEE Transactions on Smart Grid, vol. 5, no. 2, pp. 559-568, March 2014.

[16] R. Tonkoski, D. Turcotte, and T. H. M. EL-Fouly, "Impact of high pv penetration on voltage profiles in residential neighborhoods," IEEE Transactions on Sustainable Energy, vol. 3, no. 3, pp. 518-527, July 2012.

[17] EPRI, "Opendss." [Online]. Available: http://smartgrid.epri.com/SimulationTool.aspx

[18] G. Gruosso, "Analysis of impact of electrical vehicle charging on low voltage power grid," in 2016 International Conference on Electrical Systems for Aircraft, Railway, Ship Propulsion and Road Vehicles International Transportation Electrification Conference (ESARS-ITEC), Nov 2016, pp. 1-6.

[19] J. A. Jardini, C. M. V. Tahan, M. R. Gouvea, S. U. Ahn, and F. M. Figueiredo, "Daily load profiles for residential, commercial and industrial low voltage consumers," IEEE Transactions on Power Delivery, vol. 15, no. 1, pp. 375-380, 200.

[20] Z. Zhang, T. El-Moselhy, I. Elfadel, and L. Daniel, "Calculation of generalized polynomial-chaos basis functions and gauss quadrature rules in hierarchical uncertainty quantification," IEEE Trans. on Computer Aided Design of Integred Circuits and Systems, vol. 33, no. 5, pp. 728$740,2014$.

[21] A. Papoulis and U. Pillai, Probability, random variables and stochastic processes, 4th ed. McGraw-Hill, 112001.

[22] P. Pillay and M. Manyage, "Definitions of voltage unbalance," IEEE Power Engineering Review, vol. 21, no. 5, pp. 49-51, May 2001.

[23] Z. Zhang, T. A. El-Moselhy, I. M. Elfadel, and L. Daniel, "Stochastic testing method for transistor-level uncertainty quantification based on generalized polynomial chaos," IEEE Transactions on Computer-Aided Design of Integrated Circuits and Systems, vol. 32, no. 10, pp. 15331545 , Oct 2013.

[24] A. Sandu, C. Sandu, and M. Ahmadian, "Modeling multibody systems with uncertainties. part i: Theoretical and computational aspects," Multibody System Dynamics, vol. 15, no. 4, pp. 369-391, 2006.

[25] P. Maffezzoni, Z. Zhang, S. Levantino, and L. Daniel, "Variationaware modeling of integrated capacitors based on floating random walk extraction," IEEE Transactions on Computer-Aided Design of Integrated Circuits and Systems, vol. 37, no. 10, pp. 2180-2184, Oct 2018.

[26] IEEE, "Distribution test feeders," may 2015. [Online]. Available: http://ewh.ieee.org/soc/pes/dsacom/testfeeders/index.html

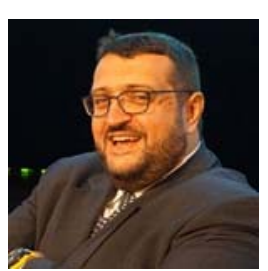

Giambattista Gruosso (M'01-SM'12) was born in 1973. He received the M.S. degrees in electrical engineering from the Politecnico di Torino, Italy, in 1999 and the Ph.D. degree in electrical engineerin from Politecnico di Torino, Italy, in 2003. From 2002 to 2011, he was a Assistant Professor with the Department of Electronics and Informatics of Politecnico di Milano. Since 2011, he has been an Associate Professor at Politecnico di Milano. His main research topics are Electrical Vehicles Transportation Electrification Electrical power systems

Optimization, and simulation of electrical systems. He is senior member IEEE and author of more than 80 papers on Journals and conferences on the topics. 


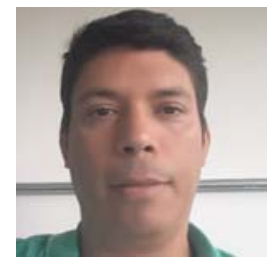

Roberto S. Netto received the Ph.D. from Universidade Federal de Itajub (UNIFEI), Institute of Electrical Systems and Energy (ISEE) in 2018. Since 2018 he has been Researcher at FIT Instituto de Tecnologia, Sorocaba, Brazil.

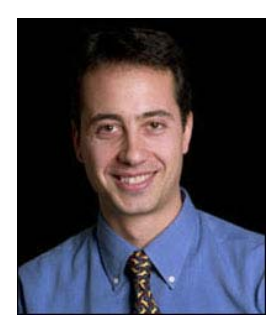

Luca Daniel (S'98-M'03-SM'19) received the Ph.D. degree in Electrical Engineering from the University of California, Berkeley, in 2003. He is currently a Full Professor in the Electrical Engineering and Computer Science Department of the Massachusetts Institute of Technology (MIT). Industry experiences include HP Research Labs, Palo Alto (1998) and Cadence Berkeley Labs (2001). His current research interests include integral equation solvers, uncertainty quantification and parameterized model order reduction, applied to RF circuits, silicon photonics, MEMs, Magnetic Resonance Imaging scanners, and the human cardiovascular system. Prof. Daniel was the recipient of the 1999 IEEE Trans. on Power Electronics best paper award; the 2003 best $\mathrm{PhD}$ thesis awards from the Electrical Engineering and the Applied Math departments at UC Berkeley; the 2003 ACM Outstanding Ph.D. Dissertation Award in Electronic Design Automation; the 2009 IBM Corporation Faculty Award; the 2010 IEEE Early Career Award in Electronic Design Automation; the 2014 IEEE Trans. On Computer Aided Design best paper award; and ten awards in conferences. Prof. Daniel recently received the 2016 Teaching Award from the MIT School of Engineering.

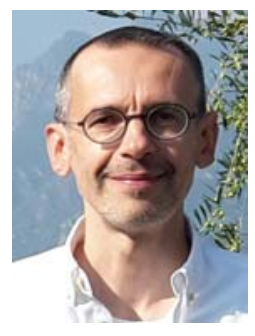

Paolo Maffezzoni (M'08-SM'15) received the Laurea degree (summa cum laude) in electrical engineering from Politecnico di Milano, Italy, in 1991 and the $\mathrm{Ph} . \mathrm{D}$. degree in electronic instrumentation from Universita' di Brescia, Italy, in 1996 . He is currently a Full Professor of electrical engineering with Politecnico di Milano, Italy. His research interests include the development of advanced computational methods for the simulation/design of nonlinear circuits and systems, oscillators, phase-domain modeling, probabilistic analysis and unconventional computing with applications to electronics and power systems. He has published about 140 research papers in international journals and conferences. He served as an Associate Editor for the IEEE Transactions on Computer-Aided Design of Integrated Circuits and Systems and the IEEE Transactions on Circuits and Systems I: Regular Papers. 